\title{
FROM MONOATOMIC MULTILAYERS TO ORDERED ALLOYS
}

\author{
J. KORECKI ${ }^{a, b}$, M. KUBIK ${ }^{a}$, N. SPIRIDIS ${ }^{b}$ AND T. ŚLĘZAK ${ }^{a}$ \\ ${ }^{a}$ Department of Solid State Physics, Faculty of Physics and Nuclear Techniques \\ University of Mining and Metallurgy, Al. Mickiewicza 30, 30-059 Kraków, Poland \\ ${ }^{b}$ Institute of Catalysis and Surface Chemistry, Polish Academy of Sciences \\ Niezapominajek, 30-239 Kraków, Poland
}

Recent progress in UHV preparation and characterization methods resulted in a large variety of novel materials. Among them, magnetic multilayers have become one of the mostly investigated system due to interesting phenomena like oscillating indirect exchange coupling, spin dependent electron transport, or large perpendicular anisotropies. An attractive possibility given by the molecular beam epitaxy is to grow the multilayer structures on atomic scale by the so-called atomic layer deposition. At the low thickness limit, a multilayer structure, in which few atomic layers of different metals are stacked alternately, is expected to be an artificial ordered alloy. Such artificial material, which does not exist in the equilibrium bulk phase, was constructed for the first time as the $A u F e$ ordered alloy of the $L 1_{0}$ structure. Our conversion electron Mössbauer spectroscopy studies of this system verified the existence of the tetragonal phase, which is responsible for the perpendicular anisotropy. The ordering process is influenced by the complicated growth of $\mathrm{Fe}$ on $\mathrm{Au}$, as shown by the atomic scale scanning tunneling microscopy investigations. Other systems to be presented are FeAl (strong ordering mechanism in the bulk) and $\mathrm{FeCr}$ (miscible in the wide concentration range) monoatomic superlattices.

PACS numbers: 75.70.Cn, 75.50.Bb, 76.80.+y

\section{Introduction}

Recent development in the epitaxial growth technique enable to control the multilayer structures on atomic scale, resulting in novel magnetic materials with such fascinating properties like perpendicular anisotropy, oscillating exchange coupling or giant magnetoresistance (GMR). Another attractive possibility of the ultrathin film technology is fabrication of metastable (often not occurring naturally) metal phases, stabilized epitaxially as for example bcc Co [1] or hcp Fe [2]. Recently, not only pure elemental phases but also artificial ordered alloys have been constructed [3] by the so-called atomic layer deposition, similar to the atomic layer epitaxy (ALE) used in the semiconductor technology. The method combines 
a layer-like structure of the constructed compound with the precisely controlled (usually by reflection high-energy electron diffraction (RHEED) oscillations) subsequent deposition of single atomic layers of the constituents. In its pure form, for semiconductors, the ALE makes use of the difference between strong chemical bonds of the compound to be grown and much weaker bonds in the pure constituent elements [4]. For metals, the situation is much more complicated and the resulting structure reflects a subtle interplay of different factors as mutual solubility of the constituents, geometrical and energetical relations defining the conditions of epitaxy, and the growth parameters (temperature and growth rate). Despite of the complicated initial conditions, it will be shown in the present studies that it is possible to fabricate ordered alloys with artificial layered structure for different metal combinations: immiscible ( $\mathrm{Au}-\mathrm{Fe})$, forming easily intermetallics $(\mathrm{Al}-\mathrm{Fe})$, and miscible in the wide concentration range ( $\mathrm{Cr}-\mathrm{Fe})$. The limitation of the investigated systems to iron compounds results from the experimental method used, which is the conversion electron Mössbauer spectroscopy (CEMS) [5]. Its exceptional role in ultrathin film structure investigation results in advantages, which are only seldom combined in a single experimental tool. Contrary to most of the surface sensitive method, CEMS has ability to probe buried layers and interfaces with the monolayer sensitivity. The method gives the atomic scale local characterization of chemical, structural, and magnetic properties simultaneously. CEMS is not only element but also isotope specific, which makes possible to perform the depth profiling of the measured parameters by the isotopic probe layer method. The concept of the ${ }^{57} \mathrm{Fe}$ probe layer can be best applied, when CEMS is combined with a fine sample preparation method, which is molecular beam epitaxy (MBE) $[6,7]$. Even monolayer resolution can be reached if a monolayer of ${ }^{57} \mathrm{Fe}$ is embedded during the preparation in a ${ }^{56} \mathrm{Fe}$ film. One has to be aware of ${ }^{57} \mathrm{Fe} /{ }^{56} \mathrm{Fe}$ interdiffusion, which may occur even at low temperature in thin film systems that grow often far from the thermal equilibrium [8].

\section{Experimental details}

The samples were grown and characterized in situ using a multichamber UHV system shown in Fig. 1. Three vacuum chambers are connected with each other and with the fast entry lock-load facility (IV) through a central transport tube (III). The samples of a typical dimension $10 \times 10 \mathrm{~mm}^{2}$ (up to five per one load), attached to universal holders, can be transferred at UHV conditions between the chambers using magnetically coupled transfer rods and the magnetically driven train inside the transport tube. The preparation chamber (I) contains a miniature MBE system, consisting of metal vapor sources and quartz monitors to control the evaporation rate. The resistively heated evaporators for $\mathrm{Fe}$ isotopes, $\mathrm{Cr}, \mathrm{Au}, \mathrm{Al}$ consist of $\mathrm{BeO}$ crucibles heated with wrap-around tungsten coils. The base pressure below $10^{-10} \mathrm{hPa}$ remains during the evaporation in a low $10^{-10} \mathrm{hPa}$ range. The quartz crystal monitors allow controlling the film thickness with an accuracy of $5 \%$ of one monolayer (ML). Certain inhomogeneity of the film thickness of about $5 \%$ due to oblique deposition cannot be avoided. The quartz calibration is verified periodically using the low angle $\mathrm{X}$-ray reflectivity measurements for specially 
prepared calibration samples. The crystallographic structure and chemical composition of the samples are monitored using four-grid LEED/AES spectrometer (low-energy electron diffraction - LEED, Auger electron spectroscopy - AES) working alternatively as LEED spectrometer for diffraction patterns recording or as retarding field electron energy analyzer in the case of Auger spectroscopy.

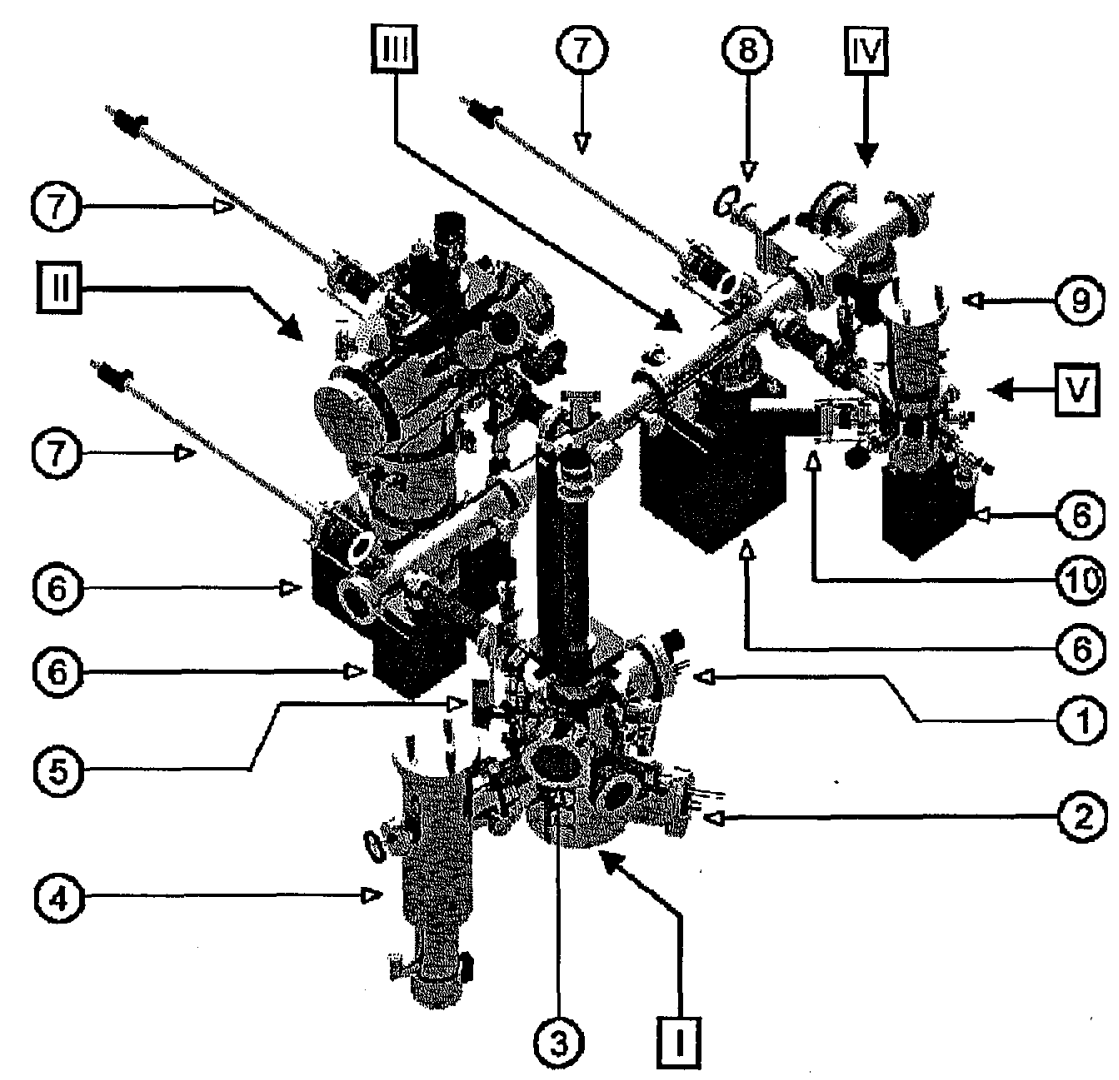

Fig. 1. The multichamber UHV system: I - growth and analysis chamber, II - chemical and thermal treatment chamber, III - central transport tube, IV - load-lock chamber, V - CEMS chamber; 1 - MBE system, 2 - titanium sublimation pump, 3 - LEED/AES spectrometer, 4 - diffusion pump with liquid nitrogen trap, 5 - quadrupole mass spectrometer, 6 - ion pump, 7 - magnetic transfers, 8 - shut-off valve, 9 - liquid nitrogen cryostat, 10 - Mössbauer transducer.

The chemical chamber (II) is especially assigned for heating the substrates and exposing the samples to the atmosphere of several gases $\left(\mathrm{O}_{2}, \mathrm{CO}\right.$, etc.). The chamber is equipped with UHV manipulator allowing heating the sample up to $1000 \mathrm{~K}$.

The CEMS chamber (V) is dedicated to the in situ measurements of the Mössbauer effect by the conversion electron detection. The sample is mounted to 
the standard holder fixed to a cold finger of a stationary liquid nitrogen cryostat. A tungsten heater and a K-type thermocouple located near the sample allow to perform the measurements in the temperature range between $80 \mathrm{~K}$ and $500 \mathrm{~K}$. A specially designed (small active area) ${ }^{57} \mathrm{Co} \gamma$ source is placed outside the UHV, inside a stainless steel tube entering the chamber. The tube is closed with a beryllium window for the $\gamma$ rays. The advantage of this solution is a small $(3 \mathrm{~cm})$ source-to-sample distance. The source is moved horizontally by a Mössbauer transducer fixed to the chamber. A large opening channeltron ( $25 \mathrm{~mm}$ diameter) is used to detect the conversion electrons resulting from the resonant absorption of the $\gamma$ radiation in the sample. To avoid a signal from the sample holder, which can be also coated with ${ }^{57} \mathrm{Fe}$ during the preparation, a movable shutter is used to limit the electrons to those, which generated in the sample. Both, the channeltron assembly and the Be-window tube can be retracted using edge-welded bellows, allowing the sample exchange.

In a separate UHV system, equipped with the LEED/AES and MBE setup, similar to that described above, the growth mode at analogous conditions can be analyzed in situ using scanning tunneling microscopy (STM) [9].

All discussed samples were prepared on cleaved $\mathrm{MgO}(001)$ substrates, however different buffer layers were used, as described below.

\section{3. $\mathbf{F e}-\mathbf{A u}$ monoatomic superlattices and single $\mathrm{Fe}$ films on $\mathrm{Au}$}

Systems with perpendicular magnetization are of great importance as potential media for magnetic recording. Such magnetic properties can be tailored by lowering dimensionality of a properly chosen system. The competing volume and surface anisotropies in a thin film often result in the uniaxial anisotropy with the easy axis perpendicular to the film plane below a certain critical thickness. This spin reorientation transition (SRT) was observed e.g. for iron films on $\mathrm{Cu}$, $\mathrm{Ru}, \mathrm{Au}, \mathrm{Pd}, \mathrm{Ag}[10,11]$. The typical value of SRT critical thickness is in the range of few atomic layers [11], which makes single films impractical for application. In multilayered systems, all interfacial features, including surface anisotropy, are enhanced and SRT may be shifted up to hundreds nanometer thickness. Perpendicular anisotropy is characteristic also of thin films of tetragonal ordered alloys [12]. Recently, layered structures occurring naturally in some ordered alloys (e.g. $L 1_{0}$-type $\mathrm{FePt}$ ) have been fabricated artificially by alternate deposition of $\mathrm{Fe}$ and $\mathrm{Pt}$ [13]. The tetragonal $L 1_{0}$ can be also constructed from single atomic layers of $\mathrm{Au}$ and $\mathrm{Fe}$ for which neither alloy nor intermetallic compounds exist in the equilibrium. Atomic distances in bcc Fe and fcc Au match within $0.6 \%$, provided that both lattices are rotated by $45^{\circ}$, so that $\mathrm{Fe}[100]$ is parallel to $\mathrm{Au}[110]$. The $\mathrm{Fe}-\mathrm{Au}$ monoatomic superlattices were investigated recently, both experimentally $[3,14]$ and theoretically $[15,16]$ and it has been demonstrated that they are an effective way to combine a high magnetic moment with a high Curie temperature and a high perpendicular interface anisotropy. From the calculations it follows that the tetragonal $L 1_{0}$ ordered $\mathrm{FeAu}$ alloy is stable with a large tetragonality. Experimentally, much lower tetragonality was observed by Takanashi et al. who documented the existence of the $L 1_{0}$ phase in the monoatomic $\mathrm{Fe}-\mathrm{Au}$ superlattices obtained by $\mathrm{MBE}$. The order parameter, derived from X-ray diffraction, was much lower 
than it could be expected from the layer-by-layer growth observed for this system but the authors pointed out that the short-range order, not seen in the diffraction experiment, is certainly much higher. The aim of our investigation was to characterize this system on atomic level for finding a reason of deterioration of ordering. The Mössbauer spectroscopy is sensitive to the local order and it should help in solving this problem.

Fe films and $\mathrm{Au}-\mathrm{Fe}$ superlattices were grown on buffer layers obtained on the $\mathrm{MgO}$ substrate in a multistage process at the typical of MBE evaporation rate of about $0.5 \mathrm{~nm} / \mathrm{min}$ [17]. First, a thin (usually $4 \mathrm{~nm}$ ) $\operatorname{Cr}(001)$ seed-layer was grown directly on $\mathrm{MgO}$ at about $100^{\circ} \mathrm{C}$, followed by a $17 \mathrm{~nm} \mathrm{Au}$ layer at $180^{\circ} \mathrm{C}$. This base layer was annealed for $1 \mathrm{~h}$ at $520^{\circ} \mathrm{C}$ and finally a $3 \mathrm{~nm} \mathrm{Au}$ layer was added at $180^{\circ} \mathrm{C}$ onto the annealed film. The resulting $(28 \times 5) \mathrm{Au}(001)$ reconstructed surface, called $\mathrm{Au}(001)$-hex, is characterized by low roughness, due to monoatomic steps only, and reconstruction ridges in two perpendicular domain directions [17]. $\mathrm{Au}(001)$-hex surface is a good template for growing flat $\mathrm{Fe}$ films $[17,18]$ for which a layer-by-layer growth mode is promoted by Au surfactant action. Atomic place exchange between $\mathrm{Fe}$ and $\mathrm{Au}$ atom occurs, leading to formation of $\mathrm{Au}$ monolayer on top of the Fe film. The mechanism of the Au surface segregation is illustrated in Fig. 2, which shows two different stages of the initial Fe growth on $\mathrm{Au}(001)$-hex observed by STM. For Fe coverages below $\approx 0.5 \mathrm{ML}, \mathrm{Fe}$ island structures are seen on the still partially reconstructed Au surface (Fig. 2a). Then, at a higher $\mathrm{Fe}$ coverage (about 0.6 ML) the character of the surface changes entirely (Fig. 2b). The reconstruction vanishes and the $\mathrm{Fe}$ islands cannot be clearly identified. The
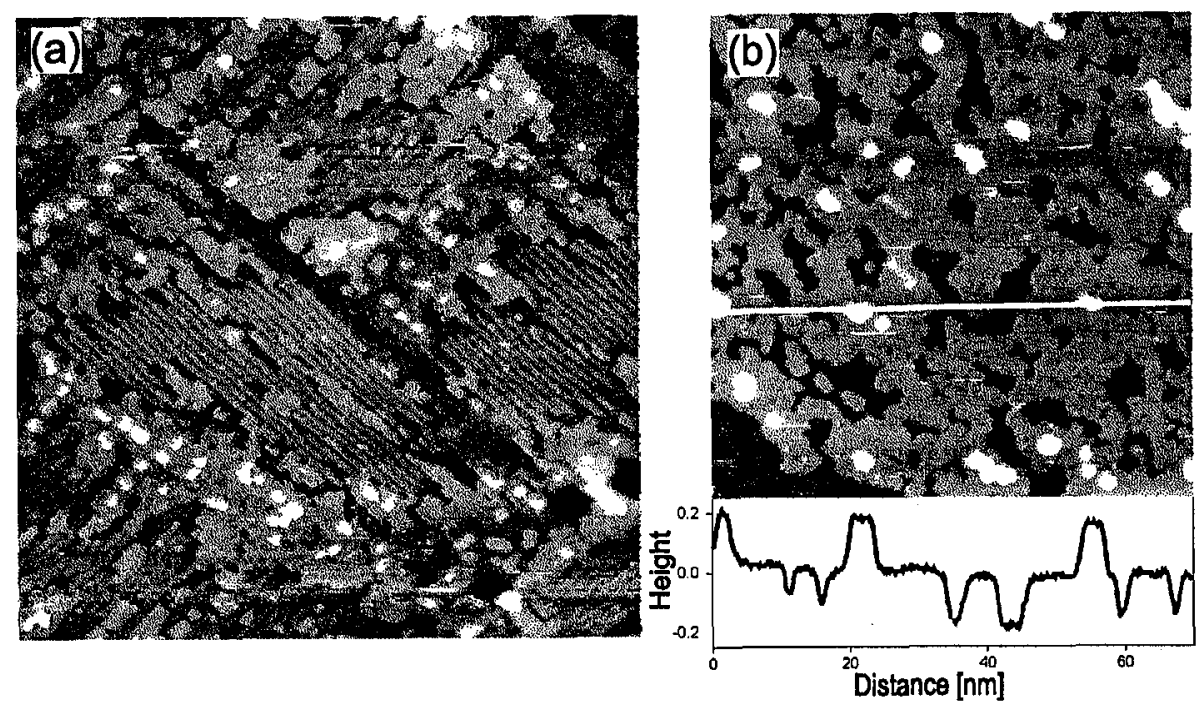

Fig. 2. Topographic STM images of different stages of Fe growth on Au(001)-hex surface: (a) $90 \times 90 \mathrm{~nm}^{2}$ scan for $0.4 \mathrm{ML} \mathrm{Fe}$ coverage, (b) $70 \times 70 \mathrm{~nm}^{2}$ scan for $0.65 \mathrm{ML}$ coverage and the depth profile along the marked line. 
vertical spacing of monoatomic steps identified in the depth profile are equally spaced by $0.2 \mathrm{~nm}$, which is very close to the value for the $\mathrm{Au}(001)$ layers. It is the strong indication that the observed pit structure, so much different from the island structure for lower coverage, is formed by $\mathrm{Au}$ atoms segregated to the surface. The vertical mass transport in this process leads inevitably to certain intermixing between $\mathrm{Au}$ and $\mathrm{Fe}$, which cannot be neglected discussing the $\mathrm{Au}-\mathrm{Fe}$ superlattice growth and properties.

When $\mathrm{Au}$ is grown on top of the $\mathrm{Fe}(001)$ film, an $\mathrm{Au}(001)$-hex surface is restored after deposition of $1 \mathrm{ML}$ Au. Thus, similar growth conditions are ensured. for subsequent layers resulting in high quality $\mathrm{Fe} / \mathrm{Au}$ superlattices.

Figure 3a presents room temperature CEMS spectra [19] of tree different superlattices denoted as $\left(\mathrm{Fe}_{n} \mathrm{~A} u_{n}\right)_{N}$ ( $n$ is the number of $\mathrm{Fe}$ or $\mathrm{Au}$ atomic layers in a single $\mathrm{Fe}-\mathrm{Au}$ sequence, $N$ is the number repetitions), for which periodic multilayer structure was proved by X-ray diffraction (XRD). For the $\left(\mathrm{Fe}_{3} \mathrm{Au}_{3}\right)_{7}$ sample the CEMS spectrum is very similar to that one for the single $3 \mathrm{ML}$ Fe film [20]. By lowering the superlattices period the CEMS spectra become very different from those of single Fe films of the corresponding thickness. The most spectacular difference comes from the increase in the Curie temperature for superlattices (for a $\mathrm{Fe}$ monolayer $T_{\mathrm{C}}$ is well below room temperature [20]). The second important modification of the magnetic properties going from single film to multilayers concerns the magnetic anisotropy. Single films above one $1 \mathrm{ML}$ are magnetized in plane [20] and for the $\mathrm{Fe}-\mathrm{Au}$ superlattices the out-of-plane component of the magnetization exists not only for the $\left(\mathrm{Fe}_{1} \mathrm{Au}_{1}\right)_{20}$ but also for the $\left(\mathrm{Fe}_{2} \mathrm{Au}_{2}\right)_{10}$ one, as can be seen from the intensity ratio of the CEMS spectra. The basic interpretation of the perpendicular anisotropy in the $\mathrm{Fe}-\mathrm{Au}$ system refers to the tetragonal distortion, which is especially large, when the ordered alloy is formed [3]. The tetragonality is clearly
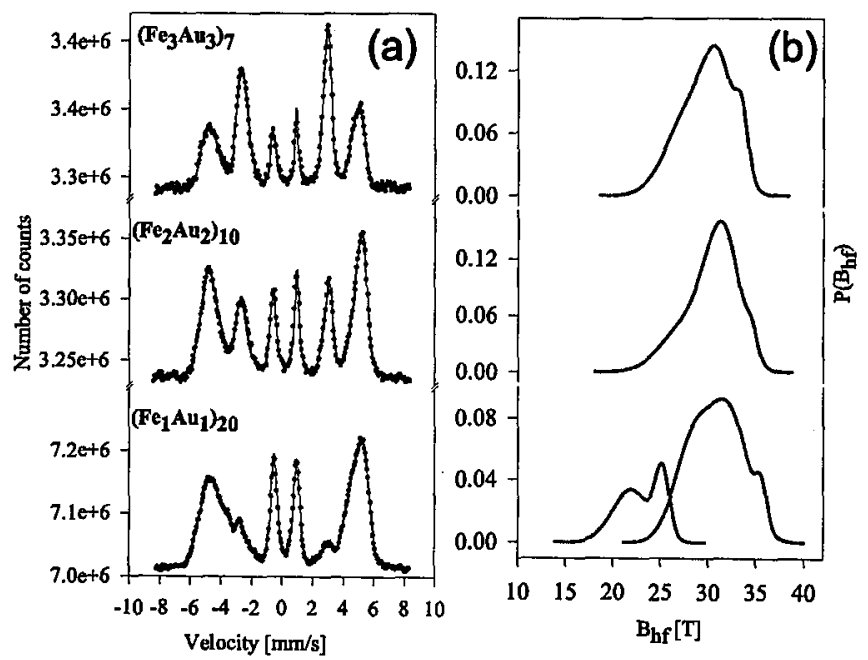

Fig. 3. Room temperature CEMS spectra of Au-Fe superlattices (a) and the distributions of the hyperfine magnetic field $B_{\mathrm{hf}}$ resulting from the numerical analysis (b). 
(a)

(b)

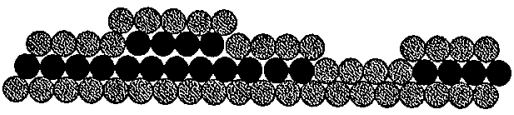

(c)
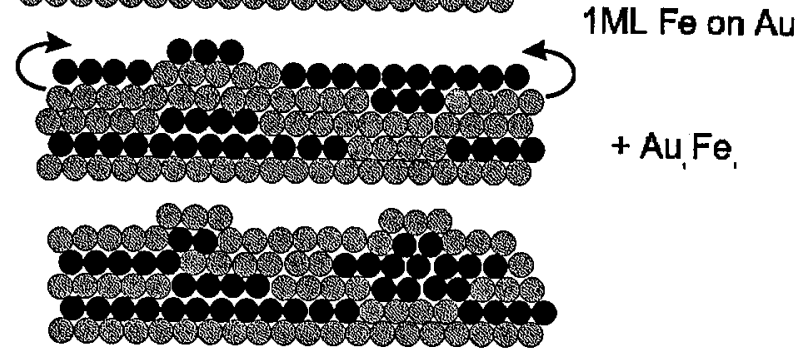

Fig. 4. The growth model of the monoatomic Au-Fe superlattice. Fe atoms are indicated in black, Au in gray. See text for details.

seen for the Fe monolayer (perpendicularly magnetized) as appearing of the spectrum component with the high quadrupole splitting [20]. Here, for the $\left(\mathrm{Fe}_{2} \mathrm{Au}_{2}\right)_{10}$ superlattice, the quadrupole splitting is negligible, so the perpendicular magnetization is supposed to come from the interface contribution to the anisotropy, as it was observed also for polycrystalline $\mathrm{Fe} / \mathrm{Au}$ multilayers [21]. In addition, the analysis of the hyperfine field distribution $B_{\mathrm{hf}}$ indicates that the contribution of the tetragonal $L 1_{0}$ order phase to the structure is much lower than expected. The tetragonal phase is characterized by a spectral component with a low hyperfine magnetic field (approximately $20 \mathrm{~T}$ ) and a high quadrupole splitting. Such component is not observed in the spectra for the $\left(\mathrm{Fe}_{3} \mathrm{Au}_{3}\right)_{7}$ and $\left(\mathrm{Fe}_{2} \mathrm{Au}_{2}\right)_{10}$ samples, for which the $B_{\mathrm{hf}}$ distribution shows a bulk-like hyperfine fields (33 T) and an interface component with a slightly enhanced $B_{\mathrm{hf}}$ (Fig. $\left.3 \mathrm{~b}\right)$. Only for the $\left(\mathrm{Fe}_{1} \mathrm{Au}_{1}\right)_{20}$ sample the $B_{\mathrm{hf}}$ distribution reveals the presence of the tetragonal phase seen from a low magnetic field $\left(\left\langle B_{\mathrm{hf}}\right\rangle=22.1 \mathrm{~T}\right.$ is very close to the theoretical value [22]) and high quadrupole splitting component. The contribution of this component is only $25 \%$, which is certainly connected with the complicated $\mathrm{Fe} / \mathrm{Au}$ growth mode, as discussed above. Assuming that the Au surface segregation takes place also after the deposition of the subsequent $\mathrm{Fe}$ monolayers, a model of the monoatomic superlattice growth is proposed in Fig. 4. After completion of the first Fe monolayer, one finds an Au monolayer on top (a). Adding the following Au monolayer results in that temporarily two Au monolayers cap the structure, the topmost of them being segregated again to the surface as soon as a Fe monolayer is added (b). The perpendicular mass transport of $\mathrm{Au}$ leads to natural aggregation of $\mathrm{Fe}$, so that the monolayer patches, in with $\mathrm{Fe}$ atoms might have locally a tetragonal symmetry, are strongly reduced $(c)$.

\section{4. $\mathrm{Fe}-\mathrm{Al}$}

Ferromagnetic (FM) films separated by a non-ferromagnetic (NM) spacer have been widely investigated because of the indirect magnetic couplings and the resulting GMR observed in these systems [23]. The indirect exchange interaction is mediated by the spin polarization of the conduction electrons via the 
Ruderman-Kittel-Kasuya-Yoshida (RKKY) interaction. The coupling is very sensitive to any structure modification but the microscopic mechanism of these phenomena remains often unexplained. Therefore, it is very important to link the resulting macroscopic properties with their microscopic origin that is reflected in the conduction electron polarization of the spacer. There are only few methods capable to measure the spacer magnetism directly. The Mössbauer spectroscopy is used rather frequently, but when a ${ }^{57} \mathrm{Fe}$ probe layer is used to study a non-magnetic spacer, the magnetic Fe atoms, embedded in the spacer, strongly modify its magnetic properties. This has motivated us to construct a non-magnetic spacer material of a layered structure that contains Fe naturally. The FeAl ordered alloy seems to be an ideal spacer material for Mössbauer studies - it is paramagnetic and contains $\mathrm{Fe}$ naturally. A strong ordering mechanism is reported for $\mathrm{Fe}$ and $\mathrm{Al}$ compounds in a wide range of their relative concentrations [24,25]. Near the 1:1 concentration, which is of our particular interest, the $\mathrm{B2}-\mathrm{CsCl}$ structure appears. The ordered alloy is for this concentration paramagnetic even at low temperatures. If a weak ferromagnetism appears, it is usually connected with defects: vacancies and $\mathrm{Fe}$ antistructure atoms (ASA). The defects disturb the environmental symmetry of the iron nucleus, which results in an electric field gradient easily detectable in the Mössbauer experiments as contributing to a quadrupole doublet [26]. The $\mathrm{FeAl}$ ordered alloy is also well suited to be obtained layer-by-layer, accordingly to its layer-like structure composed of the alternating (001) $\mathrm{Fe}$ and $\mathrm{Al}$ monolayers. If a monoatomic $\mathrm{Fe} / \mathrm{Al}$ superlattice is grown by $\mathrm{MBE}$, it is possible to change between two iron isotopes $\left({ }^{56} \mathrm{Fe}\right.$ and $\left.{ }^{57} \mathrm{Fe}\right)$, restricting the Mössbauer information to a pre-selected depth, provided that ${ }^{56} \mathrm{Fe} /{ }^{57} \mathrm{Fe}$ intermixing can be avoided.
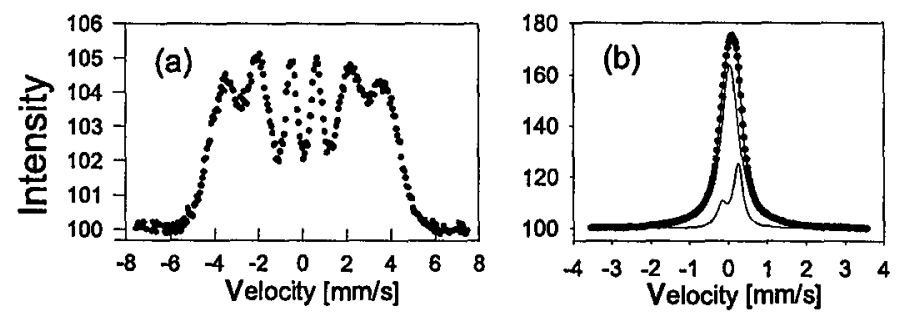

Fig. 5. Room temperature CEMS spectra of monoatomic $\left(\mathrm{Al}_{1} \mathrm{Fe}_{1}\right)_{50}$ superlattices grown on glass. (a) and $\mathrm{MgO}(001)$ (b).

By growing the $\mathrm{FeAl}$ ordered alloy, the epitaxy plays a crucial role. It can be seen from the CEMS spectra shown in Fig. 5, which are taken for two samples of the nominally same composition. A complicated spectrum of an $\left(\mathrm{Al}_{1} \mathrm{Fe}_{1}\right)_{50}$ multilayer deposited at UHV condition on a glass substrate held at room temperature (RT) (Fig. 5a) reveals a magnetic order and excludes the presence of an ordered compound. However, if the same preparation conditions were repeated for $\mathrm{MgO}(001)$ substrate, the LEED pattern indicated an epitaxial growth and from the CEMS spectrum (Fig. 5b) became evident that the grown superlattice has the $B 2$ structure of the FeAl ordered alloy. The best fit was obtained assuming two spectral components. A relatively narrow $(\mathrm{HWHM}=0.26 \mathrm{~mm} / \mathrm{s})$ single line comes 
from the ideal environments for which all nearest neighbors (nn) of $\mathrm{Fe}$ atoms are $\mathrm{Al}$ atoms. The second subspectrum, the asymmetric quadrupole doublet, is due to defects existing in the neighborhood of the iron atom [26]. A rough estimation gives the defect concentration of about $5 \%$. Recently, the FeAl monoatomic superlattices were used as non-magnetic spacers between $\mathrm{Fe}$ films and an oscillation of the conduction electron polarization vs. the spacer thickness was monitored by the CEMS studies [27].

\section{5. $\mathrm{Fe}-\mathrm{Cr}$}

In the last decade, $\mathrm{Fe}-\mathrm{Cr}$ sandwiches and multilayers are probably the most intensively studied metallic film system. The reason is their potential in device application that was opened since the antiferromagnetic coupling [28] and the GMR effect [29] was found in $\mathrm{Fe}-\mathrm{Cr}$ multilayers. Up to now the coupling mechanism is not quite clear and the alloying at the $\mathrm{Fe} / \mathrm{Cr}$ interface is strongly involved in its explanation [30]. Studying magnetic structure of $\mathrm{FeCr}$ binary compounds is then of particular interest and theoretical works use recently the approach of a hypothetical $B 2-\mathrm{FeCr}$ alloy [31, 32], resulting in unusual magnetic features in bulk [31] and at the surface [32]. B2 structure does not exist in the bulk $\mathrm{Fe}-\mathrm{Cr}$ phase diagram and it seems that it could be stabilised only artificially by epitaxial atomic layer deposition of $\mathrm{Fe}(001)$ and $\mathrm{Cr}(001)$.

$\left(\mathrm{Fe}_{1} \mathrm{Cr}_{1}\right)_{20}$ and $\left(\mathrm{Fe}_{2} \mathrm{Cr}_{2}\right)_{10}$ superlattices were deposited at the room temperature on the $20 \mathrm{~nm}$ epitaxial $\mathrm{Cr}(001)$ buffer layer grown on $\mathrm{MgO}(001)$. The LEED pattern (Fig. 6a) revealed the epitaxial growth of superlattices, however the broad spots and strong diffused background indicated the high density of defects (presumably steps). CEMS spectra taken at $80 \mathrm{~K}$ (to reduce temperature effects) are shown in Fig. 6b. Despite of a strong $\mathrm{Cr} / \mathrm{Fe}$ intermixing observed at the interfaces of $\mathrm{Cr} / \mathrm{Fe} / \mathrm{Cr}$ sandwiches [33] the CEMS spectra show that the local structure in
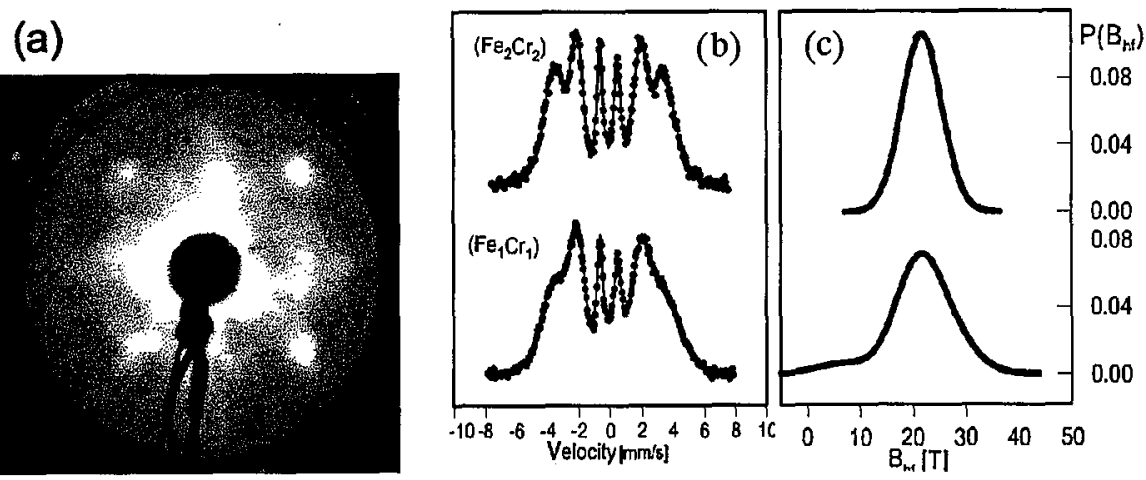

Fig. 6. (a) LEED pattern from the surface of the $\left(\mathrm{Fe}_{1} \mathrm{Cr}_{1}\right)_{20}$ superlattice grown at the room temperature on the $\mathrm{Cr}(001) / \mathrm{MgO}(001)$ substrate, and (b) $80 \mathrm{~K}$ CEMS spectra for $\left(\mathrm{Fe}_{1} \mathrm{Cr}_{1}\right)_{20}$ and $\left(\mathrm{Fe}_{2} \mathrm{Cr}_{2}\right)_{10}$ superlattices with (c) the distributions of the hyperfine magnetic field resulting from the numerical analysis. 
$\left(\mathrm{Fe}_{1} \mathrm{Cr}_{1}\right)_{20}$ and $\left(\mathrm{Fe}_{2} \mathrm{Cr}_{2}\right)_{10}$ superlattices is different, as seen from different hyperfine distributions, also shown in Fig. 6b, which reflect local coordination of the Fe atoms. Apparently, the layer sequence during the deposition is pertained to some extent in the resulting structure. The analysis of the local structure in bulk $\mathrm{FeCr}$ alloys [34] is based on a model relating the $B_{\mathrm{hf}}$ value at a Fe site with the number of $\mathrm{Cr}$ nearest and next nearest neighbors. If the model is applied to perfectly ordered superlattices, the calculated hyperfine magnetic field at the $\mathrm{Fe}$ site in the $\mathrm{Fe}_{2} \mathrm{Cr}_{2}$ superlattice exceeds that one in the $\mathrm{Fe}_{1} \mathrm{Cr}_{1}$ superlattice by about $5 \mathrm{~T}$. For the real samples, only the average $B_{\mathrm{hf}}$ can be compared, giving for both superlattices nearly the same values. A possible explanation of the discrepancy is an enhancement of the $\mathrm{Cr}$ magnetic moment in the partially ordered $\mathrm{Fe}_{1} \mathrm{Cr}_{1}$ superlattice, in accordance with the theoretical prevision for the $B 2-\mathrm{FeCr}$ alloy [31].

\section{Conclusions}

Studying of monoatomic superlattices becomes important for basic understanding of magnetic multilayers and for constructing of novel materials with unusual magnetic features. By the epitaxial growth at the room temperature, far from the thermodynamic equilibrium, ordered alloy phases can be stabilized. The Mössbauer spectroscopy showed up to be an effective tool of probing their local structural and magnetic properties.

This work was supported by the University of Mining and Metallurgy under grant No. 11.11.220.01.

\section{References}

[1] J. Dekoster, E. Jedryka, M. Wojcik, G. Langouche, J. Magn. Magn. Mater. 126, 12 (1993).

[2] M. Maurer, M. Piecuch, M.F. Ravet, J.C. Ousset, J.P. Sanchez, C. Aaron, J. Dekoster, D. Raoux, A. Andres, M. De Santis, A. Fontaine, F. Baudelet, J.L. Rouviere, B. Dieny, J. Magn. Magn. Mater. 93, 15 (1991).

[3] K. Takanashi, S. Mitani, M. Sano, H. Fujimori, H. Nakajima, A. Osawa, Appl. Phys. Lett. 67, 1016 (1995).

[4] M.A. Herman, H. Sitter, Molecular Beam Epitaxy - Fundamentals and Current Status, 2nd ed., Springer-Verlag, Berlin 1996.

[5] K. Nomura, Y. Ujihira, A. Vertes, J. Radioanal. Nucl. Chem. Artic. 202, 103 (1996).

[6] J. Korecki, U. Gradmann, Phys. Rev. Lett. 55, 2491 (1985).

[7] J. Prokop, M. Przybylski, T. Ślęzak, J. Korecki, Surf. Rev. Lett. 4, 1239 (1997).

[8] M. Przybylski, U. Gradmann, J. Korecki, J. Magn. Magn. Mater. 69, 199 (1987).

[9] J. Korecki, N. Spiridis, B. Handke, J. Prokop, J. Haber, Electron Technol. 29, 269 (1996).

[10] C. Liu, S.D. Bader, J. Vac. Sci. Technol. A 8, 2727 (1989).

[11] R.P. Cowburn, L. Ferre, J.-P. Jamet, S.J. Gray, J.A.C. Bland, Phys. Rev. B 55, 11593 (1997).

[12] A. Cebollada, D. Weller, J. Sticht, G.R. Harp, R.F.C. Farrow, R.F. Marks, R. Savoy, J.C. Scott, Phys. Rev. B 50, 3419 (1994). 
[13] S. Mitani, K. Takanashi, M. Sano, H. Fujimori, A. Osawa, H. Nakajima, J. Magn. Magn. Mater. 148, 163 (1995).

[14] S. Riedling, N. Knorr, C. Mathieu, J. Jorzick, S.O. Demokritov, B. Hillebrands, R. Schreiber, P. Grünberg, J. Magn. Magn. Mater. 198-199, 348 (1999).

[15] Z.P. Shi, J.F. Cooke, Z. Zhang, B.M. Klein, Phys. Rev. B 54, 3030 (1996).

[16] J.T. Wang, Z.Q. Li, Q. Sun, Y. Kawazoe, J. Magn. Magn. Mater. 183, 42 (1998).

[17] N. Spiridis, J. Korecki, Appl. Surf. Sci. 141, 313 (1999).

[18] V. Blum, Ch. Rath, S. Müller, L. Hammer, K. Heinz, J.M. Garcia, J.E. Ortega, J.E. Prieto, O.S. Hernan, J.M. Gallego, A.L. Vasquez de Parga, R. Miranda, Phys. Rev. B 59, 15966 (1999).

[19] T. Ślęzak, W. Karaś, M. Kubik, M. Mohsen, M. Przybylski, N. Spiridis, J. Korecki, Hyperfine Interact. C 3, 409 (1998).

[20] J. Korecki, M. Kubik, N. Spiridis, T. Ślęzak, to be published.

[21] S. Honda, K. Koguma, M. Nawate, I. Sakamoto, J. Appl. Phys. 82, 4428 (1997).

[22] G.Y. Guo, H. Ebert, Hyperfine Interact. 97/98, 11 (1996).

[23] Ultrathin Magnetic Structures I, II, Eds. B. Heinrich, J.A.C. Bland, Springer, Berlin 1994.

[24] Yu.V. Kudryavtsev, V.V. Nemoshkalenko, Y.P. Lee, K.W. Kim, J. Appl. Phys. 82, 5043 (1997).

[25] J.E. Frackowiak, Hyperfine Interact. 54, 793 (1990).

[26] R. Wagoner, M. Reissner, W. Steiner, J. Bogner, H. Sassik, P. Pongratz, B. Sepiol, J. Magn. Magn. Mater. 140-144, 57 (1995).

[27] T. Ślęzak, M. Kubik, J. Korecki, J. Magn. Magn. Mater. 198-199, 405 (1999).

[28] P. Grunberg, R. Schreiber, Y. Pang, M.B. Brodsky, H. Sowers, Phys. Rev. Lett. 57, 2442 (1986).

[29] M.N. Baibich, J.M. Broto, A. Fert, F. Nguyen Van Dau, F. Petroff, P. Etienne, G. Creuzet, A. Friederich, J. Chazelas, Phys. Rev. Lett. 61, 2472 (1988).

[30] A. Davies, J.A. Stroscio, D.T. Pierce, R.J. Cellota, Phys. Rev. Lett. 76, 4175 (1996).

[31] S.L. Qiu, P.M. Marcus, V.L. Moruzzi, Phys. Rev. B 58, 2651 (1998).

[32] H. Bouzar, M. Benakki, M. Zemirli, A. Mokrani, C. Demangeat, H. Dreysse, Surf. Sci. 381, 117 (1997).

[33] M. Kubik, M. Przybylski, T. Ślęzak, to be published.

[34] S.M. Dubiel, J. Żukrowski, J. Magn. Magn. Mater. 15-18, 655 (1980). 